\title{
A NEW PROOF THAT NILPOTENT GROUPS ARE CCR
}

\author{
J. M. G. FELL
}

Introduction. It has recently been shown by Dixmier [2], and also by Kirillov [7], that a connected nilpotent Lie group $G$ is CCR. This means that, if $T$ is any irreducible unitary representation of $G$ and $f \in L_{1}(G), T_{f}$ is a completely continuous operator in the space of $T$. In this note we give a new proof of this fact.

The methods of Dixmier and Kirillov actually yield more than complete continuity. They are able to single out a dense subspace of $L_{1}(G)$ whose image under any irreducible unitary representation of $G$ consists of operators with trace. Our proof does not give this extra information; on the other hand it requires considerably less analysis. It is based on Rosenberg's theorem [11], which asserts that a separable $C^{*}$-algebra which has (to within equivalence) only one irreducible ${ }^{*}$-representation must be ${ }^{*}$-isomorphic with the algebra of all completely continuous operators in some Hilbert space. In terms of the hull-kernel topology of the dual space $\hat{A}$ of an arbitrary $C^{*}$-algebra $A$, Rosenberg's result may be stated thus: An element $T$ of $\hat{A}$ is completely continuous (i.e., $T_{a}$ is completely continuous for all $a$ in $A$ ) if and only if the one-element set $\{T\}$ is closed in $\hat{A} .{ }^{1}$ The problem is thus reduced to that of showing that the dual space of a connected nilpotent Lie group is $T_{1}$ (i.e., points are closed). This is done by an inductive procedure which, apart from the topological preliminaries, is a special case of Takenouchi's procedure in [12].

In this paper, by a representation of a locally compact group $G$ (or *-algebra $A$ ) we always mean a unitary representation (or a *-representation with no null space) acting in a separable Hilbert space. If $G$ is a locally compact group, $C^{*}(G)$ will be its group $C^{*}$-algebra (the completion of $L_{1}(G)$ under the minimal regular norm); we use the same letter for a representation of $G$ and the corresponding representation of $C^{*}(G)$. A representation $T$ of $G$ is completely continuous if $T_{a}$ is completely continuous for all $a$ in $C^{*}(G)$. If $N$ is a closed normal subgroup of $G$, and $T^{\prime}$ is a representation of $G / N$, the representation $T$ of $G$ given by $T_{x}=T_{N x}^{\prime}$ is said to be lifted from $T^{\prime}$; if $T^{\prime}$ is completely continuous, so is $T$.

Received by the editors January 3,1961.

1 The latter form of Rosenberg's theorem is to be found explicitly in [6, Theorem 4]. Indeed, Rosenberg's theorem is easily seen to be a very special case of Theorem 1 of [6]. 
Let $K$ be a closed subgroup of $G$. If $T$ is a representation of $G$, $T \mid K$ is the restriction of $T$ to $K$; if $J$ is a family of representations of $G, J \mid K$ is the set of all $T \mid K$, where $T \in J$. If $T$ is a representation of $K, U^{T}$ is the representation of $G$ induced from $T$ (see [8]); if $J$ is a family of representations of $K, U^{\mathfrak{J}}$ is the set of all $U^{T}$, where $T \in \mathfrak{J}$.

Let $A$ be a $C^{*}$-algebra (or a locally compact group). As in [3], if $\mathcal{S}$ and $\mathcal{J}$ are two families of representations of $A$, we say that $\delta$ is weakly contained in $J$ if every positive functional on $A$ associated with any representation in $S$ is a weak $*$ limit of sums of positive functionals associated with representations in $\mathfrak{J}$ (or, if every function of positive type on $A$ associated with any representation in $S$ is a uniform-on-compacta limit of sums of functions of positive type associated with representations in $J$ ). If each is weakly contained in the other, $S$ and $J$ are weakly equivalent. Now assume that $A$ is separable. Then $\hat{A}$, the dual space of $A$, is defined as the family of all (equivalence classes of) irreducible representations of $A$, equipped with the so-called hull-kernel topology. Restricted to $\hat{A}$, the relation of weak containment coincides with closure in the hull-kernel topology of $\hat{A}$.

For more information on the hull-kernel topology, and the relations of weak containment and weak equivalence, we refer the reader to [3].

1. Orbits in the dual space of a normal subgroup. In this section we fix a separable locally compact group $G$ with unit element $e$, and a closed normal subgroup $K$ of $G$ which (a) is of Type I and has smooth dual, ${ }^{2}$ and (b) is regularly embedded in $G .^{3}$ Let us review the known facts about the relationship between $\hat{K}$ and $\hat{G}$ (see [10]).

If $L$ is any representation of $K$ and $x \in G, L^{x}$ will be the representation of $K$ defined by: $L_{\xi}^{x}=L_{x \xi x-1}$. If $L \in \hat{K},\left\{x \in G \mid L^{x} \cong L\right\}$ is a closed subgroup of $G$ containing $K$, called the stationary subgroup of $L$ and denoted by $S_{L}$. An orbit is a subset of $\hat{K}$ of the form $\left\{L^{x} \mid x \in G\right\}$ (for some $L$ in $\hat{K}$ ). Each orbit is a Borel set (with respect to the Borel structure of $\hat{K}$ defined in [9]). Now let $T$ be an element of $\hat{G}$. The restriction of $T$ to $K$ uniquely determines a projection-valued measure $P_{T}$ on the Borel subsets of $\hat{K}$, whose values are projections in the space $H(T)$ of $T$. By the regular embeddedness of $K, P_{T}$ is confined to some orbit $\theta$ (i.e., $P_{T}(\hat{K}-\theta)=0$ ); we say that $T$ is associated with $\theta$, and refer to this $\theta$ as $\theta_{T}$. Let $\hat{G}_{\theta}$ be the set of all $T$ in $\hat{G}$ which are asso-

2 For the notion of smooth dual, see $[9$, p. 151]. It is now known (see [6]) that $A$ is of Type $I$ if and only if it has a smooth dual; so one of these hypotheses may be omitted.

By this we mean that the quotient Borel space of the orbits of $\hat{K}$ under $G$ is countably separated. Mackey in [10] gives a formally weaker definition (see p. 302). 
ciated with the orbit $\theta$; more generally, if $M \subset \hat{K}$ and $M$ is a union of orbits, $\hat{G}_{M}$ will be the set of all $T$ in $\hat{G}$ which are associated with some orbit contained in $M$. A classification of $\hat{G}_{\theta}$ is afforded by the following fact:

Proposition $1 .{ }^{4}$ Let $\theta$ be an orbit, and $L$ an element of $\theta$ with stationary subgroup $S_{L}$. Then the mapping $W \rightarrow U^{W}$ sets up a one-to-one correspondence between the family $Q_{L}$ of all elements $W$ of $\left(S_{L}\right)^{\wedge}$ whose restriction to $K$ is a multiple of $L$, and the family $\hat{G}_{\theta}$.

Now consider the topologies of $\hat{K}$ and $\hat{G}$. Though the fact will not be needed here, we observe that the Borel structure of $\hat{K}$ is just that generated by the topology of $\hat{K}$. Indeed, $K$ being of Type I, the elements of $K$ (considered as representations of $C^{*}(K)$ ) are determined by their kernels (see [6, Theorem 1]); so we may invoke Theorem 4.1 of [4] to obtain the desired conclusion.

For each $x$ in $G$, the map $L \rightarrow L^{x}$ is a homeomorphism of $\hat{K}$ onto itself; hence the closure in $\hat{K}$ of a union of orbits is again a union of orbits. More generally, we have the following easy lemma:

Lemma 1. The map $(x, L) \rightarrow L^{x}$ is continuous on $G \times \hat{K}$ into $\hat{K}$.

The following proposition was established in the course of the proof of Theorem 4.3 of [5]:

Proposition 2.5 If $H$ is any closed subgroup of $G$ containing $K$, and $T$ is any representation of $H$, then $U^{T} \mid K$ is weakly equivalent to the set of all $(T \mid K)^{x}(x \in G)$.

From this together with Proposition 1 we deduce immediately:

LEMma 2. If $\theta$ is an orbit and $T \in \hat{G}_{\theta}$, then $T \mid K$ is weakly equivalent to $\theta$.

Lemma $3{ }^{6}$ If $M$ is a closed subset of $\hat{K}$ which is a union of orbits, then $\hat{G}_{M}$ is closed in $\hat{G}$.

Proof. Suppose $T$ is an element of $\hat{G}$ weakly contained in $\hat{G}_{M}$. By [3, Corollary of Theorem 1.3], $T \mid K$ is weakly contained in $\hat{G}_{M} \mid K$. By Lemma 2, this implies that $\theta_{T}$ is weakly contained in $M$, i.e., $\theta_{T} \subset \bar{M}=M$. So $T \in \hat{G}_{M}$.

4 This is Theorem 8.1 of [10], for the case of the trivial multiplier.

- For this proposition $K$ need not be of Type I or regularly embedded.

- The stronger statement that, if $M$ is a union of orbits, the closure of $G_{M}$ is $G_{\bar{M}}$ seems likely to be true, but may require a good deal more technical apparatus for its proof. 
Leмma $4 .^{7}$ Let $\theta$ be an orbit and $L$ an element of $\theta$; and suppose that the following three conditions hold: (i) $\theta$ is closed in $\hat{K}$; (ii) $S_{L}$ is normal; (iii) the natural map $x S_{L} \rightarrow L^{x}$ of $G / S_{L}$ onto $\theta$ is a homeomorphism of $G / S_{L}$ into $\hat{K}$. Then the one-to-one correspondence $W \leftrightarrow U^{W}$ between $Q_{L}$ and $\hat{G}_{\theta}$ (see Proposition 1) is a homeomorphism (with respect to the topologies of $\left(S_{L}\right)^{\wedge}$ and $\hat{G}$ relativized to $Q_{L}$ and $\hat{G}_{\theta}$ respectively).

Proof. Theorem 4.1 of [5] asserts that the map $W \rightarrow U^{W}$ is continuous. To show that its inverse is continuous, we suppose that $\mathfrak{M} \subset Q_{L}, N \in Q_{L}$, and $U^{\mathfrak{N}}$ weakly contains $U^{N}$, and show that $\mathfrak{T}$ weakly contains $N$.

Since $U^{\mathscr{T}}$ weakly contains $U^{N}, U^{\mathfrak{T}} \mid S_{L}$ weakly contains $U^{N} \mid S_{L}$. Hence, by Proposition 2 applied to the closed normal subgroup $S_{L}$, the orbit of $N$ (in $\left.\left(S_{L}\right)^{\wedge}\right)$ is contained in the closure of the union $V$ of the orbits of the elements of $\mathfrak{M}$. In particular $N \in \bar{V}$. Hence there is a net $\left\{\left(M^{\nu}\right)^{x^{\nu}}\right\}$ of elements of $V$ such that $M^{\nu} \in \mathscr{N}, x^{\nu} \in G$, and

$$
\left(M^{\nu}\right)^{x^{\nu}} \rightarrow N \text { in }\left(S_{L}\right)^{\wedge} \text {. }
$$

Since $N$ and $M^{v}$ are in $Q_{L}, N \mid K$ is a multiple of $L$, and $\left(M^{v}\right)^{x^{v}} \mid K$ is a multiple of $L^{x^{\nu}}$. Hence, restricting (1) to $K$ (and using [3, Corollary of Theorem 1.3]), we deduce that $L^{x^{\nu}} \rightarrow L$. By condition (iii) of the lemma, this shows that $x S_{L} \rightarrow S_{L}$ (in $G / S_{L}$ ); so we might as well have assumed from the beginning that $x^{\nu} \rightarrow e$. But then, by (1) and Lemma

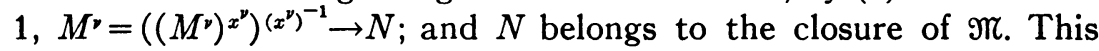
completes the proof.

Let $A$ be any $C^{*}$-algebra. If $T$ is a representation of $A$, its range $B=T(A)$ is again a $C^{*}$-algebra; and we refer to the dual space $\hat{B}$ of $B$ as the dual space of $T$. If $T$ is a representation of a locally compact group $G_{0}$, the dual space of $T$ is the dual space of the representation of $C^{*}\left(G_{0}\right)$ which corresponds to $T$.

Proposition 3. Let $A$ be either a separable $C^{*}$-algebra or a separable locally compact group, and $T$ an element of $\hat{A}$. The dual space of $T$ is homeomorphic with the closure of $\{T\}$ in $\hat{A}$ (with the topology relativized from $\hat{A})$. The representation $T$ is completely continuous if and only if $\{T\}$ is closed in $\hat{A}$, i.e., if and only if the dual space of $T$ is a oneelement set.

Proof. We need consider only the case that $A$ is a separable $C^{*}$ algebra. The first statement of the proposition follows immediately from the definition of the hull-kernel topology. The second statement follows from Rosenberg's theorem [11] (see the Introduction).

Returning to our fixed group $G$, we now have:

\footnotetext{
7 The proof of Lemma 4 actually does not require condition (i).
} 
THEOREM 1. Let $\theta$ be an orbit in $\hat{K}$, and $L$ an element of $\theta$; and suppose that the conditions (i), (ii), (iii) of Lemma 4 hold. Then, for each $W$ in $Q_{L}, W$ and $U^{W}$ have homeomorphic dual spaces. In particular, $W$ is completely continuous if and only if $U^{W}$ is.

Proof. Let $W$ be in $Q_{L}$. Since $\theta$ is closed, $\hat{G}_{\theta}$ is closed in $\hat{G}$ by Lemma 3; so by Proposition 3 the dual space of $U^{W}$ is the closure of $\left\{U^{W}\right\}$ relative to $\hat{G}_{\theta}$. Again, by conditions (i) and (iii) of Lemma 4, $\{L\}$ is closed in $\hat{K}$. Hence, using [3, Corollary of Theorem 1.3], we find that $Q_{L}$ is closed in $\left(S_{L}\right)^{\wedge}$; so the dual space of $W$ is the closure of $\{W\}$ relative to $Q_{L}$. Now, by Lemma $4, Q_{L}$ and $\hat{G}_{\theta}$ are homeomorphic under the correspondence $M \leftrightarrow U^{M}$. So $W$ and $U^{W}$ have homeomorphic dual spaces.

The last statement of the theorem follows immediately from the first together with Proposition 3.

2. Nilpotent groups. Let $G$ be a simply connected nilpotent Lie group of dimension $n, \mathfrak{g}$ its Lie algebra, and $E$ the exponential mapping of $g$ into $G$. The following facts about $G$ are well known (see for example $[1] ;[12])$ :

(1) $E$ is one-to-one onto $G$.

(2) Every closed connected subgroup of $G$ is simply connected.

(3) The center of $G$ is connected.

(4) If $\mathfrak{h}$ is a subalgebra of $\mathfrak{g}, E(\mathfrak{h})$ is the connected closed subgroup of $G$ (normal if $\mathfrak{h}$ is an ideal) whose Lie algebra is $\mathfrak{h}$.

(5) $g$ has a composition series:

$$
\{0\}=g_{0} \subset g_{1} \subset \cdots \subset g_{r}=\mathfrak{g},
$$

where $\mathfrak{g}_{i}$ is an ideal of $\mathfrak{g}$, each $\mathfrak{g}_{i} / \mathfrak{g}_{i-1}$ is one-dimensional, and $\left[\mathfrak{g}, \mathfrak{g}_{i}\right]$ $\mathrm{Cg}_{\mathfrak{g}_{i-1}}$. In particular, the center of $g$ contains $\mathfrak{g}_{1}$.

THEOREM 2. Every irreducible unitary representation of $G$ is completely continuous.

Proof. This will be proved by induction in the dimension $n$ of $G$. For $n=1$ the theorem is trivial. So let $n$ be greater than 1 ; and assume the theorem true for all connected nilpotent groups of dimension less than $n$.

Suppose first that the center $C$ of $G$ is of dimension greater than 1. If $T$ is an irreducible unitary representation of $G, T \mid C$ is the identity operator multiplied by a character $\chi$ of $C$. Since $\operatorname{dim} C \geqq 2$, there is a one-dimensional closed subgroup $K$ of $C$ on which $\chi \equiv 1 ; T$ will then be lifted from an irreducible unitary representation $T^{\prime}$ of $G / K$. Since $G / K$ is nilpotent, connected, and of dimension less than $n, T^{\prime}$ 
is completely continuous by the inductive hypothesis. Hence so is $T$.

So it is enough to assume that $\operatorname{dim} C=1$, i.e., $C=E\left(g_{1}\right)$. Let $G_{2}=E\left(g_{2}\right) ; G_{2}$ is a closed normal Abelian subgroup of $G$, isomorphic with the two-dimensional vector group. Suppose that $\{u, v\}$ is a basis of $\mathfrak{g}_{2}$ with $u \in \mathfrak{g}_{1}$. Since $\left[\mathfrak{g}, \mathfrak{g}_{2}\right] \subset \mathfrak{g}_{1}$, there is a real linear functional $\lambda$ on $g$ such that

$$
[x, v]=\lambda(x) u \quad(x \in \mathfrak{g}) .
$$

Since $v$ is not in the center of $\mathfrak{g}, \lambda$ is not identically zero. Let $\chi_{a, b}$ ( $a, b$ real) be the character of $G_{2}$ sending $E(r u+s v)$ into $e^{i(a r+b s)}$; for $\xi$ in $G$ and $\eta$ in $G_{2}$, let $\chi_{a, b}^{\xi}(\eta)=\chi_{a, b}\left(\xi \eta \xi^{-1}\right)$. If $\xi=E(x), x \in g$, we verify that

$$
\chi_{a, b}^{\xi}=\chi_{a, \lambda(x) a+b} .
$$

Thus the orbit of $\chi_{a, b}$ under $G$ consists of (i) $\theta_{b}^{0}=\left\{\chi_{0, b}\right\}$ if $a=0$, (ii) $\theta_{a}^{1}$ $=\left\{\chi_{a, b} \mid b\right.$ real $\}$ if $a \neq 0 . \cdot$ In particular, $G_{2}$ is regularly embedded in $G$ and all orbits are closed in $\hat{G}_{2}$. Further, by (3), the stationary subgroup of $\chi_{a, b}$ is $G$ if $a=0$, and $S=E(N)$, where $N$ is the null space of $\lambda$, if $a \neq 0$. It is easy to see that $N$ is an ideal of $g$ (in fact, it contains all Lie products $[x, y]$, where $x, y \in g$ ); so $S$ is closed, simply connected, and normal, and of dimension less than $n$. For fixed $a \neq 0$, the correspondence $E^{\prime}(x+N) \rightarrow \chi_{a, \lambda(x) a+b}$ ( $E^{\prime}$ being the exponential mapping of $\mathrm{g} / N$ onto $G / S)$ is clearly a homeomorphism of $G / S$ onto $\boldsymbol{\theta}_{a}^{\mathbf{1}}$. Thus each orbit in $\hat{G}_{2}$ satisfies all the conditions of Theorem 1 .

Now let $T$ be any irreducible unitary representation of $G$. Since $G_{2}$ is regularly embedded in $G, T$ is associated with some orbit in $\hat{G}_{2}$.

Case I. $T$ is associated with $\theta_{a}^{1}, a \neq 0$. By Proposition $1, T$ is of the form $U^{W}$, where $W \in \hat{S}$. Since $S$ is a connected nilpotent group of dimension $n-1$, the inductive hypothesis assures us that $W$ is completely continuous. Hence, by Theorem $1, T$ is also completely continuous.

Case II. $T$ is associated with $\theta_{b}^{0}, b$ real. This means that $T$ restricted to $G_{2}$ is the identity operator times the character $\chi_{0, b}$. In particular, $T$ is the identity operator on $C$. It follows that $T$ is lifted from an irreducible unitary representation $T^{\prime}$ of $G / C$, which is of dimension $n-1$. By the inductive hypothesis $T^{\prime}$ is completely continuous; hence so is $T$. This completes the proof.

\section{BiBLIOGRAPHY}

1. J. Dixmier, L'application exponentielle dans les groupes de Lie résolubles, Bull. Soc. Math. France 85 (1957), 113-121.

2. - Sur les représentations unitaires des groupes de Lie nilpotents. V, Bull. Soc. Math. France 87 (1959), 65-79. 
3. J. M. G. Fell, The dual spaces of $C^{*}$-algebras, Trans. Amer. Math. Soc. 94 (1960), 365-403.

4. - C C*algebras with smooth dual, Illinois J. Math. 4 (1960), 221-230.

5. - Weak containment and induced representations of groups, Canad. J. Math. (to appear).

6. J. G. Glimm, Type I $C^{*}$-algebras, Ann. of Math. (2) 73 (1961), 572-612.

7. A. A. Kirillov, On unitary representations of nilpotent Lie groups, Dokl. Akad. Nauk SSSR 130 (1959), 966-968.

8. G. W. Mackey, Induced representations of locally compact groups. I, Ann. of Math. 55 (1952), 101-139.

9. - Borel structure in groups and their duals, Trans. Amer. Math. Soc. 85 (1957), 134-165.

10. - Unitary representations of group extensions. I, Acta Math. 99 (1958), 265-311.

11. Alex Rosenberg, The number of irreducible representations of simple rings with no minimal ideals, Amer. J. Math. 75 (1953), 523-530.

12. O. Takenouchi, Sur la facteur-représentation d'un groupe de Lie résoluble de Type (E), Math. J. Okayama Univ. 7 (1957), 151-161.

UNIVERSITY OF WASHINGTON

\section{ON A RESULT OF BAER}

\section{MAXWELL ROSENLICHT}

The theorem is that if $H$ and $N$ are subgroups of the group $G$, with $N$ normal in $G$, and if the set of commutators

$$
\left\{h n h^{-1} n^{-1} \mid h \in H, n \in N\right\}
$$

is finite, then so is the group $[H, N]$ generated by these. Here we give a proof that seems considerably simpler than the original one [1] (cf. also [2, exposé 3] for the case where both $H$ and $N$ are normal in $G$ ).

The most important special case of Baer's result concerns a group $G$ whose center is of finite index, in which case the assertion is that the commutator group of $G$ is finite. Here is a brief proof, similar to the one given in $[3$, p. 59]: It suffices to show that any product of commutators of elements of $G$ can be written as such a product with at most $n^{3}$ factors, $n$ being the index of the center of $G$. Noting that there are at most $n^{2}$ distinct commutators, and that in any product of commutators any two factors may be brought together by replacing the intermediate factors by conjugates, also commutators, it suffices to show that the $(n+1)$ th power of a commutator is the product of $n$

\footnotetext{
Received by the editors December 15, 1960.
} 